\title{
Bone Mineral Density in Diabetes Mellitus Foot Patients for Prediction of Diabetic Neuropathic Osteoarthropathic Fracture
}

\author{
Jae-Ho Cho', Tae-Hong Min², Dong-II Chun², Sung-Hun Won², Su Yeon Park ${ }^{3}$ Kwonwoo Kim ${ }^{4}$, Young Yi ${ }^{5}$ \\ 'Department of Orthopaedic Surgery, Chuncheon Sacred Heart Hospital, Hallym University, Chuncheon; \\ ${ }^{2}$ Department of Orthopaedic Surgery, Soonchunhyang University Seoul Hospital, Soonchunhyang University College of Medicine, Seoul; \\ ${ }^{3}$ Department of Biostatistics, Soonchunhyang University Seoul Hospital, Soonchunhyang University College of Medicine, Seoul; \\ ${ }^{4}$ Department of Health Administration, Sejong Public Health Center, Sejong; \\ ${ }^{5}$ Department of Orthopaedic Surgery, Seoul Foot and Ankle Center, Inje University Seoul Paik Hospital, Inje University College of Medicine, \\ Seoul, Korea
}

Corresponding author

Young Yi

Department of Orthopaedic Surgery, Seoul

Foot and Ankle Center, Inje University Seoul

Paik Hospital, Inje University College of

Medicine, 9 Mareunnae-ro, Jung-gu, Seoul

04551, Korea

Tel: +82-2-2270-0023

Fax: +82-2-2270-0236

E-mail:20vvin@naver.com

Received: July 13, 2020

Revised: August 19, 2020

Accepted: August 20, 2020
Background: Diabetic neuropathic osteoarthropathy (DNOAP) is known as debilitating diabetes complications. The aim of study is to compare bone mineral density (BMD) among diabetic foot and DNOAP, and investigate the impact of BMD proceeded from diabetic foot to DNOAP.

Methods: A DNOAP group (subgroup A and subgroup B) and control group were examined for this study. Subgroup A $(n=21)$ were patients diagnosed with DNOAP with the development of new foot and ankle fractures, whereas subgroup $B(n=4)$ were patients being managed with the diabetic foot before a diagnosis of DNOAP. BMD was also evaluated before the diagnosis. Control group $(n=30)$ was diabetic foot patients without DNOAP. The demographic data, clinical and radiologic data, comorbidities, and BMD were compared for each group. And optimal BMD score was reviewed to predict fractures in neuropathic arthropathy.

Results: BMD was significantly lower in DNOAP group (group A and B) compared with control group. Also neuropathic arthropathy group showed poor radiological results. After comparisons of 2 group lumbar and femur BMD was significantly different, but logistic regression analysis revealed that low femur T-score could be risk predictors of the condition. Base on the data of group B and control group, the cut-off point for predicting foot and ankle fracture-related with DNOAP was -1.65 of femur BMD.

Conclusions: Low BMD shows greater incidence in foot and ankle fracture patients associated with neuropathic arthropathy. A femur T score can be a risk predictor of diabetic neuropathic arthropathy for diabetic foot patients.

Key Words: Arthropathy · Bonedensity · Diabetic foot · Neurogenic

\section{INTRODUCTION}

Diabetic neuropathic osteoarthropathy (DNOAP) is a destructive process of a denervated joint. The bony components and DNOAP can be frequently noticed with foot and ankle fractures. Although it was initially associated with tertiary syphilis, diabetes is the main cause of neuropathic arthropathy in the developed
Mineral Research

This is an Open Access article distributed under the terms of the Creative Commons Attribution Non-Commercial License (https://creativecommons.org/licenses/by-nc/4.0/) which permits unrestricted non-commercial use, distribution, and reproduction in any medium, provided the origina work is properly cited.

\section{KSBMR}


countries. The pathophysiology of this joint destroying process is not well known, but is considered to be multifactorial, and neuropathy seems to be binding.[1-4] The presence of dense peripheral sensory neuropathy, abnormal circulation, and history of preceding trauma seems to be the primary risk factors for this possibly limb-threatening deformity.[5]

All patients with diabetes have an increased risk for fractures, and it has been suggested that the presence of peripheral osteopenia may play a role.[6] Furthermore, foot deformities, prior amputations, joint infections, or surgical trauma may result in excessive stress that can lead to diabetic neuropathic arthropathy.[7] The neurotraumatic theory shows that loss of protective sensation can lead to repeated microtrauma, which can make DNOAP. There is also the neurovascular theory, which suggests that when there is a high flow vascular state in affected patients, the increased blood flow washes out local calcium in bone and can create localized osteopenia.[8]

Osteoporosis has also been hypothesized to have a role in DNOAP development, but it is still controversial.[9,10] Current concepts suggest that osteopenic bones show more initial microfracture, and there is evidence that both neuropathy and diabetes are related with osteopenia.[11] Diabetic induced neuropathy causes bone dissolution and increases osteoclast activity by increasing the expression of receptor activator of nuclear transcription factor $\beta$ ligand, which can lead to increased activation of nuclear transcription factor $\beta$.[12] This osteolysis pathway can further be enhanced by the presence of free radicals, hyperlipidemia, increased ambient glucose concentration and advanced glycation end-products present in diabetic patients.[13] These things contribute to the fracture risk in osteoporosis.
Since most diabetic neuropathic arthropathy is diagnosed when suffering from painless fracture or destructive deformity of the joint, we can deduce that bone mineral density (BMD) will be significantly lower in DNOAP patients with foot and ankle fracture than in diabetic patients without neuropathic arthropathy. When deformity progresses or fractures occur, diabetic neuropathic arthropathy patients require surgical treatment, but it is very important to predict the course of the disease in advance because of the high infection rate and reoperation rate.[14]

This study configures the hypothesis: The diabetic neuropathic arthropathy precedes diabetic foot. And diabetic neuropathic arthropathy patients show significantly lower BMD levels than diabetic foot patients.

The aim of study is twofold:

(1) to compare BMD among diabetic foot and diabetic neuropathic arthropathy patients;

(2) to investigate the impact of BMD on the progression of diabetic foot into diabetic neuropathic arthropathy

\section{METHODS}

\section{Study group selection}

This retrospective study was evaluated at our institution and approved by the Inje University Seoul Paik Hospital Institutional Review Board (PAIK2018-08-007). In this study, patients with BMD among patients diagnosed with DNOAP and diabetic foot were selected from our university hospitals from March 2012 to December 2018.

In this study, 2 subgroups from 25 DNOAP patients were examined in a cross-sectional study design (Fig. 1). The mean diabetic neuropathic arthropathy patient group age at the time of diagnosis was 70.8 years (range, 63-88 years), and

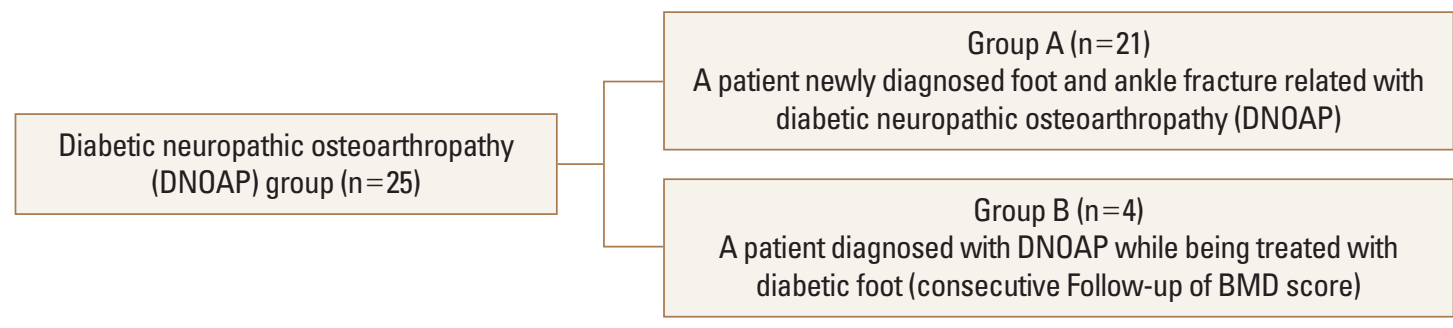

Control group $(n=30)$

A diabetic foot patient with neuropathy

Fig. 1. Demonstration of patient group and control group. BMD, bone mineral density. 
the minimum follow-up period was 14.2 months. Subgroup $A(n=21)$ was a new patient who was diagnosed with foot and ankle fracture-related with DNOAP. Total 15 patients who were non-plantigrade foot or recurrent history of ulceration or fracture were treated with surgical treatment, while the other 6 case is treated by conservative treatment such as non-weight bearing and cast protection. Patients who underwent surgical treatment were confirmed through clinical features, radiography, and pathology. Patients who received non-surgical treatment were diagnosed through characteristic fracture patterns and clinical features of the ankle or Lisfranc joints that occur without external impact.

Subgroup B $(n=4)$ was a patient diagnosed with DNOAP while already being treated with diabetic foot. Diagnosis was based on clinical features including unilateral redness, swelling, and a skin temperture $>2^{\circ} \mathrm{C}$ compared with the contralateral foot using an infrared thermometer. The group $B$ patients have consecutive BMD score follow-up data from diabetic foot to DNOAP. The control group was composed of 30 diabetic foot patients with neuropathy without evidence of DNOAP. The average age of the group was 71 years (range, 65-87 year).

\section{Measurement}

Foot and ankle weight bearing radiographs were obtained from all patients. The talo-1st metatarsal angle and calcaneal pitch were measured in the weight-bearing foot lateral view, and the talo-1st metatarsal angle and navicular coverage angle were measured in the weight-bearing foot anteroposterior (AP) view. In the weight-bearing ankle AP view, an ankle fracture or progression of ankle arthritis were compared. Clinical workup was compared by using Short Form-36 (SF-36) in a cross-sectional manner.[15]

All subjects underwent full medical history examination (with emphasis on presence or absence of diabetic complications) and thorough clinical examination including a full assessment of the diabetic foot. Laboratory investigations were done for all subjects, which included fasting blood sugar, postprandial blood sugar, $\mathrm{HbA} 1 \mathrm{c} \%$, calcium, phosphorus, and alkaline phosphatase.

A dual energy X-ray absorptiometry (DXA) scanner (ELunar Inc., Madison, WI, USA) was used for scanning. The whole body, lumbar spine, and unilateral hip were scanned. The T-score and Z-score were registered. The World Health Organization definitions of osteopenia and osteoporosis were used osteopenia $(-2.5<\mathrm{T}<-1)$ and osteoporosis $(\mathrm{T} \leq$ -2.5).[16] The Z-score was calculated according to a normal reference (incorporated in the scanner) for age, weight, sex, and ethnic matched material. In 4 cases, BMD values obtained from ellipses were adopted, and the results of the same DXA scanner were also confirmed to be taken.

\section{Statistical analysis}

We compared the demographic finding, clinical and radiologic findings, laboratory data, comorbidities as well as the BMD between the DNOAP group and control group. Parametric data were represented as mean \pm standard deviation (SD), while nonparametric data were represented as the median and interquartile range. Differences between the 2 groups were detected using the student's $t$-test for parametric data and Mann-Whitney $\mathrm{U}$ test for nonparametric data.

We allocated each patient's T-score into three categorical groups; normal, osteopenia, osteoporosis. After yielding each group's odds ratio, the $P$-value was measured. The cutoff point for the decrease of BMD that allowed the prediction of DNOAP was determined by receiver operating characteristic (ROC) curve analysis. This analysis was performed using the last BMD measured in diabetic foot management before DNOAP diagnosis in group $B$ patients, and they are evaluated with a control group. Cut-off points were selected based on the maximal sensitivity and specificity sum. For all tests, $P$-values of less than 0.05 were considered significant. Statistical analyses were performed using the software package SPSS for Windows version 16.0 (SPSS Inc., Chicago, IL, USA).

\section{Power analysis}

The effect size for the difference in the BMD (femoral T score) between the group B and control group was 4.31, which is calculated using the means and SDs of BMD group $A$ and control group (-1.87 and -0.34 in both groups, respectively). The effect size of 4.31 indicated that a minimum sample size of 2 in each group would be required to detect the difference with $80 \%$ power at a $5 \%$ significance level. Given an anticipated dropout rate of $20 \%$, a total of 3.2 patients was required in each group. Therefore, the sample size in group $B(n=4)$ was minimally satisfied to demonstrate that such a difference existed. 
Table 1. Characteristic of diabetic neuropathic arthropathy group and control group (diabetic foot with neuropathy patient group)

\begin{tabular}{|c|c|c|}
\hline Variables & DNOAP group $(n=25)$ & Control group $(n=30)$ \\
\hline \multicolumn{3}{|l|}{ Diabetes type } \\
\hline Type 1 & 5 & 8 \\
\hline Type 2 & 20 & 22 \\
\hline Diabetes duration (yr) & 16.4 & 10.1 \\
\hline \multicolumn{3}{|l|}{ Diabetes treatment } \\
\hline Insulin & 8 & 9 \\
\hline Oral hypoglycemic & 12 & 18 \\
\hline Diet and exercise & 1 & 3 \\
\hline No treatment & 4 & 0 \\
\hline \multicolumn{3}{|l|}{ Charcot stage } \\
\hline Stage 1 & 6 & \\
\hline Stage 2 & 15 & - \\
\hline Stage 3 & 3 & - \\
\hline Unkown & 1 & - \\
\hline \multicolumn{3}{|l|}{ Charcot locational } \\
\hline Lisfranc & 16 & - \\
\hline Hindfoot & 4 & - \\
\hline Forefoot & 7 & - \\
\hline Ankle & 8 & - \\
\hline \multicolumn{3}{|l|}{ Wagner classification } \\
\hline Grade 1/2 & - & 21 \\
\hline Grade 3 & - & 7 \\
\hline Grade 4/5 & - & 2 \\
\hline
\end{tabular}

The data is presented as number.

${ }^{a}$ Some feet had more than one site of involvement.

DNOAP, diabetic neuropathic osteoarthropathy.

\section{RESULTS}

Total 25 patients of DNOAP group and 30 patients of control group were included in this study. And diabetes duration was 16.4 years in DNOAP and 10.1 years in control group. Oral hypoglecimic was the most common diabetic treatment in both groups. In the DNOAP group, the charcot stage 2 was most common, and Lisfranc joint was most common location. In the control group, Grade 1 or 2 was the most marked in Wagner classification (Table 1).

Table 2. Patients' demographics

\begin{tabular}{lcc}
\hline & $\begin{array}{c}\text { DNOAP group } \\
(\mathrm{n}=25)\end{array}$ & $\begin{array}{c}\text { Control group } \\
(\mathrm{n}=30)\end{array}$ \\
\hline Age (yr) & $70.8(63$ to 88$)$ & 71 (65 to 87) \\
Sex & 9 & 11 \\
Male & 16 & 19 \\
Female & $26.9 \pm 6.32$ & $27.2 \pm 5.94$ \\
BMl & & \\
Comorbidity & 24 & 30 \\
Hypertension & 10 & 15 \\
Ischemic heart disease & 4 & 4 \\
Retinopathy & 7 & 6 \\
Nephropathy & 12 & 15 \\
Peripheral arterial disease & & \\
\hline
\end{tabular}

The data is presented as mean \pm standard deviation, median (range), or number.

DNOAP, diabetic neuropathic osteoarthropathy; BMI, body mass index.
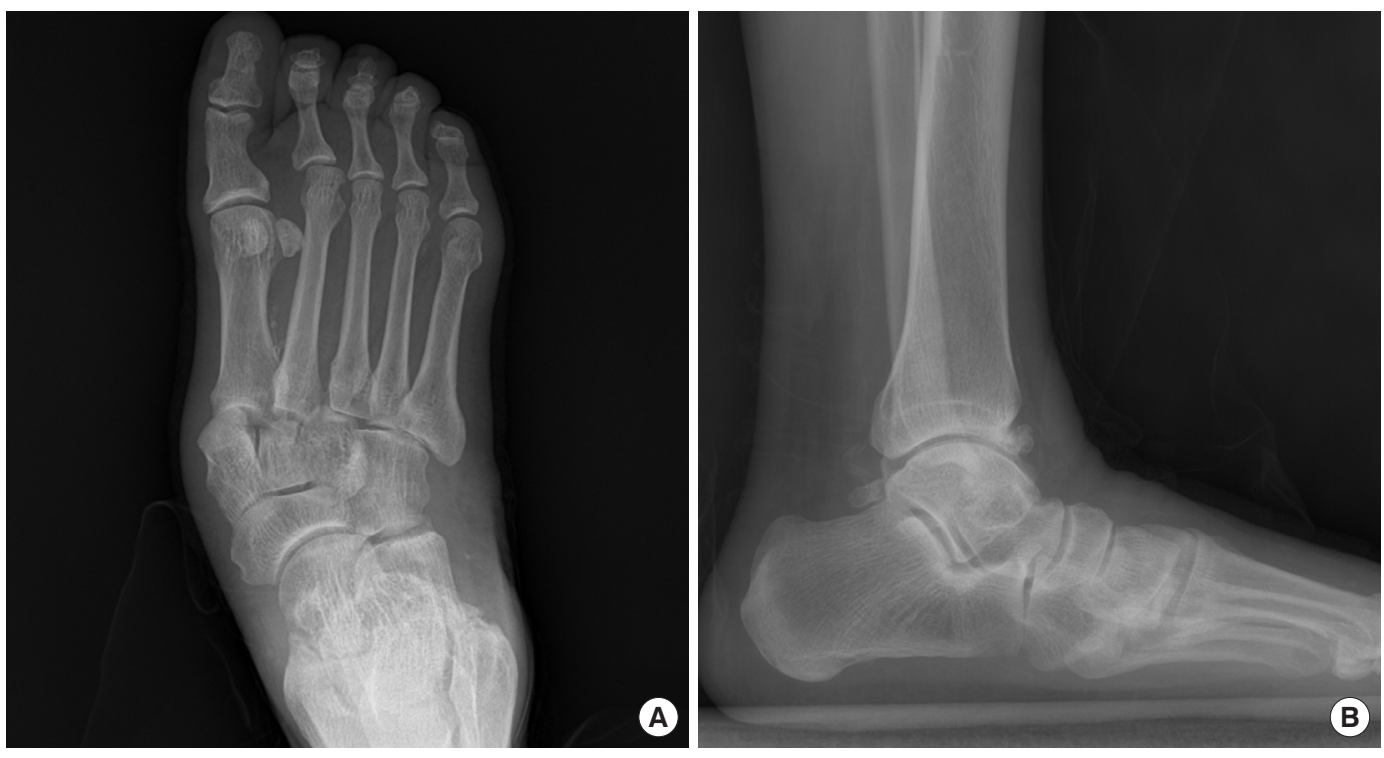

Fig. 2. Radiographic findings of diabetic neuropathic osteoarthropathy. (A) Weight bearing foot anteroposterior view. (B) Weight bearing foot lateral view. 
All patients with DNOAP were diagnosed after lower limb fracture or deformity (Fig. 2). Subgroup B patients were found while undergoing diabetic foot management. In the DNOAP group, diabetic and other complications follow, 24 patients showed hypertension disease, 10 ischemic heart disease (IHD) patients, 4 retinopathy patients, 7 nephropathy patients, and 12 peripheral arterial disease (PAD) patients. And for the control group, 30 patients with hypertension and other diabetic complications such as IHD patients, 4 retinopathy patients, 6 nephropathy patients and 15 patients with PAD. The DNOAP patient group and control group did not show significant differences in patient demographics such as age, sex, and body mass index (BMl; Table 2).

The DNOAP group showed poor radiological results. In

Table 3. Comparison of radiographic and clinical result between diabetic neuropathic arthropathy group and control group

\begin{tabular}{lccc}
\hline & $\begin{array}{c}\text { DNOAP } \\
\text { group }\end{array}$ & $\begin{array}{c}\text { Control } \\
\text { group }\end{array}$ & $\begin{array}{c}P \text { - } \\
\text { value }\end{array}$ \\
\hline Radiographic result & & & \\
Foot lateral view & & & \\
$\quad$ Lateral talo-1st metatarsal angle & $17.5 \pm 6.4$ & $3.3 \pm 4.9$ & $<0.01$ \\
Calcaneal pitch & $16.3 \pm 3.6$ & $22.8 \pm 4.7$ & $<0.01$ \\
Weight bearing foot anteroposterior view & & \\
AP talo-1st metatarsal angle & $11.5 \pm 5.8$ & $15.9 \pm 4.1$ & $<0.05$ \\
Navicular coverage angle & $22.3 \pm 6.7$ & $10.4 \pm 4.2$ & $<0.01$ \\
Clinical result & & & \\
SF-36 & & & \\
PCS & $31.7 \pm 8.5$ & $32.4 \pm 3.2$ & NS \\
PCS ag & $34.3 \pm 9.1$ & $36.7 \pm 4.8$ & NS \\
MCS & $42.8 \pm 12.6$ & $52.5 \pm 16.3$ & $<0.05$ \\
MCS ag & $40.1 \pm 12.7$ & $51.4 \pm 17.3$ & $<0.05$ \\
\hline
\end{tabular}

The data is presented as mean \pm standard deviation.

DNOAP, diabetic neuropathic osteoarthropathy; AP, anteroposterior; SF36, short form-36; PCS, physical component summary; MCS, mental component summary; ag, age- and gender-adjusted; NS, not significant. the weight-bearing foot lateral view, both the lateral talo1st metatarsal angle and calcaneal pitch were significantly lower in the DNOAP group $(P<0.01)$, and in the weightbearing foot AP view, the AP talo-1st metatarsal angle and navicular coverage angle were also meaningfully lower compared to the control group $(P<0.05)$. SF-36 level appeared low to the diabetic neuropathic group in all sections except pain $(P<0.05$; Table 3$)$. There was no statistically significant difference regarding descriptive data and laboratory data between both groups (Table 4).

DNOAP group showed a significantly lower median lumbar T score and Z score compared with control group. However, the femur T score showed significantly different in the logistic regression analysis (Table 5 ).

The ROC curve showed that femur T score was useful to determine cut-off values for DNOAP ( $P<0.05 ;$ Fig. 3$)$. The area under the curve for femur T score was 0.755 (0.589$0.921)$, and the appropriate cut-off value was -1.65 .

Table 4. Laboratory results of diabetic neuropathic arthropathy group and control group

\begin{tabular}{lccc}
\hline & DNOAP group & Control group & $P$-value \\
\hline SBP & $135.8 \pm 15.5$ & $134.7 \pm 14.9$ & 0.57 \\
DBP & $82.2 \pm 9.8$ & $82.6 \pm 8.8$ & 0.54 \\
FBS & $156.4 \pm 43.5$ & $151.3 \pm 48.8$ & 0.18 \\
2hrpp & $208.2 \pm 62.1$ & $204.7 \pm 63.3$ & 0.63 \\
HbA1c & $8.82 \pm 1.1$ & $8.79 \pm 1.32$ & 0.81 \\
Ca & $9.5 \pm 0.6$ & $9.2 \pm 0.6$ & 0.38 \\
Po4 & $4.12 \pm 0.8$ & $4.13 \pm 0.9$ & 0.82 \\
ALP & $220.8 \pm 85.7$ & $227.8 \pm 79.6$ & 0.88 \\
\hline
\end{tabular}

The data is presented as mean \pm standard deviation. Stands for $P<0.01$ highly significant.

DNOAP, diabetic neuropathic osteoarthropathy; SBP, systolic blood pressure; DBP, diastolic blood pressure; FBS, fasting blood sugar; 2hrpp, 2-hour post-prandial; HbA1c, hemoglobin A1c; Ca, calcium; P04, phosphate; ALP, alkaline phosphatase.

Table 5. Comparisons of bone mineral density between diabetic neuropathic osteoarthropathy group and diabetic foot with neuropathy

\begin{tabular}{|c|c|c|c|c|}
\hline & DNOAP group & Control group & $P$-value & $P$-value (OR $[95 \% \mathrm{Cl}])^{\mathrm{b})}$ \\
\hline \multicolumn{5}{|l|}{ T score } \\
\hline Spine 2-5 & $-1.77(\mathrm{IQR}=1.98)$ & $-1.14(\mathrm{IQR}=2.11)$ & 0.023 & 0.428 (1.12 [0.99 to 1.27]) \\
\hline Femur & $-1.96(\mathrm{IQR}=1.72)$ & $-0.34(\mathrm{IQR}=2.55)$ & $<0.001$ & $<0.05$ (1.96 [1.59 to 2.44]) \\
\hline \multicolumn{5}{|l|}{ Z score } \\
\hline Spine 2-5 & $-1.11(\mathrm{IQR}=2.27)$ & $-0.71(\operatorname{IOR}=2.12)$ & 0.024 & 0.372 (1.05 [0.94 to 1.18]) \\
\hline Femur & $-1.48(I \mathrm{IQR}=2.08)$ & $-0.69(I Q R=2.46)$ & 0.022 & 0.185 (1.04 [0.84 to 1.30]) \\
\hline
\end{tabular}

Stands for $P<0.05$ significant.

${ }^{a}$ Comparison of both group using Mann-Whitney test. ${ }^{b}$ Logistic regression analysis

DNOAP, diabetic neuropathic osteoarthropathy; IOR, interquartile range; $\mathrm{OR}$, odds ratio; $\mathrm{Cl}$, confidence interval. 


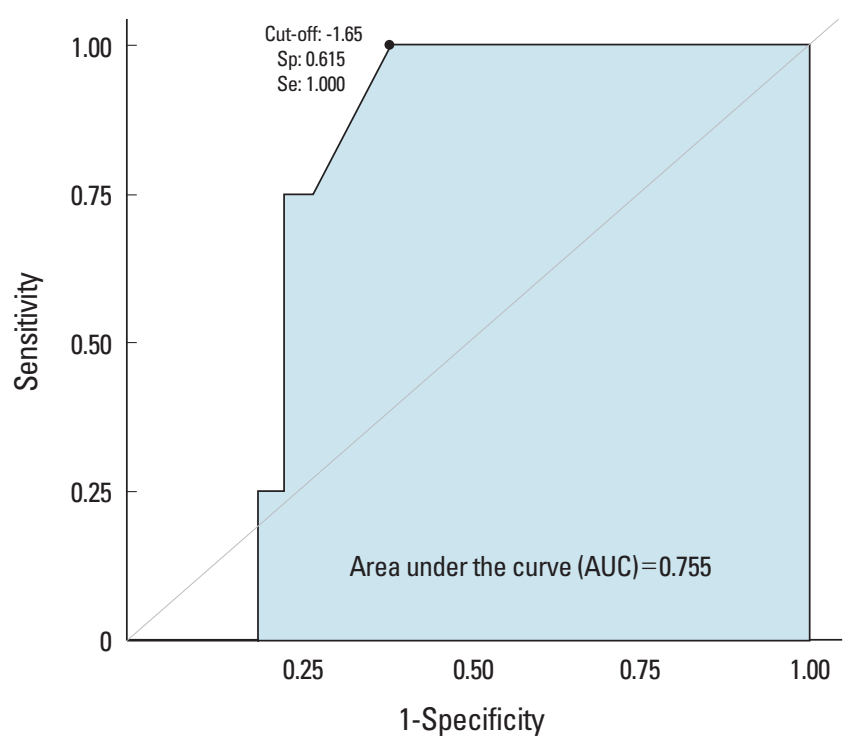

Coordinates of ROC curve (optimal cut-off point)

\begin{tabular}{lcccccc}
\hline & Threshold & Sensitivity & Specificity & Accuracy & ppv & npv \\
\hline pre & -1.65 & 1 & 0.6154 & 0.6667 & 0.2857 & 1 \\
\hline
\end{tabular}

Fig. 3. Receiver operator characteristic curve of femur T-score to predict diabetic neuropathic osteoarthropathy for diabetic foot patient with neuropathy.

\section{DISCUSSION}

Osteoporosis has been considered as a major risk factor in the development of DNOAP.[14] There is evidence that a higher risk of fractures can happen to the patients suffering from diabetes mellitus.[17] Mixed with the frequent fractures in DNOAP foot, this has led to the idea that low BMD may affect neuropathic arthropathy development. $[2,17]$ Reduced peripheral, but not axial BMD, has been indicated in patients with neuropathic arthropathy. $[9,13,17]$ A causal relationship between neuropathic arthropathy and regional osteopenia has been considered. And recent$l y$, diabetes is the main cause of neuropathic arthropathy. The purpose of this paper is to figure out whether BMD is significantly lower in DNOAP patients with fractures at foot and ankle levels, and a chance of it as risk predictor.

Analysis of BMD and neuropathic osteoarthropathy patients was done in previous studies.[10,18,19] There are 2 methods for measuring BMD of DNOAP: central BMD and calcaneal BMD measurement. Although calcaneal BMD has the benefit that it is been measured at a location adjacent to the progression of DNOAP disease, it reflects limited osseous demineralization of the peripheral part, various errors may occur due to patient specificity. Therefore, central BMD measurement is more meaningful than the cacaneal BMD, and also since changes according to DNOAP tend to show general osteoporosis in patients, this study performed central BMD measurement. Furthermore, the previous investigations of BMD and DNOAP in patients with diabetes have compared patients with a control group that consisted of healthy volunteers but have not compared patients with DNOAP with a control group of patients with diabetes mellitus (DM) without neuropathy. Until now, progression of DM foot patients with neuropathy to DNOAP was unclear. There are multiple factors, but it is common for the patient to be diagnosed because of fractures in the lower limb. This paper is meaningful because comparison of DNOAP patients and DM foot patients with neuropathy were done, and also this is the first study to present $B M D$ values for prediction of DNOAP.

In this study, at least one foot and ankle fracture was accompanied in all DNOAP patients, and clear medial foot arch disappearance was confirmed. It should be noted that patients with this pattern are more likely to develop additional fractures or progress to osteomyelitis, which can lead to amputation. Therefore, as the study result suggests, Low 
femoral T score in patients with DNOAP group with foot and ankle fracture requires careful monitoring in diabetic foot patients with neuropathy.

On the other hand, there are many reports that the reduction of BMD is significant in diabetic patients.[6] In the control group, the mean BMD was lower than the age average in both T-score and Z-score. However, even lower BMD was observed in the DNOAP group. It is thought to be pronounced deficiency in systemic BMD rather than sitespecific among the neuropathic arthropathy fracture groups reported by Herbst et al. [14]. It is predicted that while progression of the diabetic foot to DNOAP. Systemic changes occur which lead to neuropathic osteoarthropathy.

There have been various studies analyzing risk factors in DNOAP. Age is a debated but associated risk factor with DNOAP. Petrova et al. [20] reported the Charcot neuropathy typically presents in patients with diabetes during age between fifties and sixties. Other studies report the most patients with DNOAP in their sixth and seventh decades. [21,22] Fauzi et al. [23] and Nóbrega et al. [24] reported that age below 60 and 55 years were significant risk factors for DNOAP individually. Most studies showed the ratio of DNOAP in men and women was approximately the same and difference by sex is not definite.[25] However, Nehring et al. [26] reported that male gender was more likely to suffer from DNOAP. Whether weight is associated with increased risk of DNOAP was yet to be argued. Some studies reported positive correlations.[26-28] However, no association was found in other studies between DNOAP and a BMI of more than $25 \mathrm{~kg} / \mathrm{m}^{2} .[23,29]$

Most studies confirmed that the diabetes duration was a risk factor for DNOAP occurrence.[23,25,29] However, the demographic features of patients with type 1 and type 2 diabetes developing the DNOAP showed type differences. [16] Patients with type 1 had a longer duration of diabetes than those with type 2, but developed Charcot at a younger age. Petrova et al. [20] included 83 patients, 44 with type 1 diabetes presented DNOAP mostly between 40 to 49 year, while 41 type 2 patients in 50 to 59 years; however, the type 1 patients developed DNOAP with a significantly longer duration than those with type 2 diabetes $(24 \pm 8.4$ vs. $13 \pm 8.1$ years). Pakarinen et al. [27] reported the average duration of type 1 was 28 years, and that of type 2 was 14 years for 36 patients,

The relationship between BMD and DNOAP is not well known. It is unknown whether regional osteopenia is a result of the inflammatory process that accompanies the bone injury or is a risk factor for neuropathic joint disorders developments. Jones and Wolf [30] reported primary resorption of bone without subluxation, dislocation, and/or fracture in the active Charcot process.

Nonetheless, osteopenia, reduced bone stiffness, and decreased BMD in acute and chronic DNOAP patients have been confirmed radiographically.[14,20] Sinacore et al. [31] reported that inflammation in DNOAP might contribute to or exacerbate a rapid loss of BMD. The relation between BMD loss and immobilization or off-loading is still in question. Some studies reported the BMD might further decrease during casting and no weight bearing.[20] However, Pakarinen et al. [27] reported that for patients with acute DNOAP after 6 months of treatment, immobilization and offloading does not lead to marked disuse osteoporosis. BMD of DNOAP is also different according to the pattern of initial destruction. Herbst et al. [14] divided 61 Charcot feet or ankles into three subtypes, fracture pattern, dislocation pattern, and combined fracture-dislocation pattern, and reported that the fracture pattern was associated with a peripheral deficiency of $B M D$, while the dislocation pattern was not. El Oraby et al. [32] analyzed the effects of BMD and PAD to the diabetes patients and DNOAP patients, focusing on type II DM patients. In this study, female gender, femur T score, and PAD were analyzed as significant risk factors. It showed that the femur T score was a significant risk factor for DNOAP, which is the same as this study. However, this paper was conducted to both type I and type II DM patients, and DM foot patients with neuropathy was chosen to be the control group instead of DM patients. Also, an optimal range through BMD analysis before DNOAP occurred is provided. Therefore, the importance of $\mathrm{BMD}$ monitoring in diabetic foot patients with neuropathy in the disease process can be explained in more detail.

In this study, femur T score -1.65 was calculated as optimal threshold in diabetic foot patients with neuropathy. This suggests that DM foot patients with neuropathy need more strict BMD management. Usually, under score of -1.0 is diagnosed as osteopenia and osteoporosis drugs is required, but in the DM foot patient with neuropathy,[33] and we should consider active osteoporosis drugs from T score below -1.65 to prevent progression of DM foot to DNOAP.

Our study has several drawbacks. First, the sample size 
of Group B sample size is small. This is because people who measured BMD within a year before DNOAP occurs were few. On the other hand, the overall DNOAP group is 25 cases, and it is hard to say that the number of DNOAPs is small because the occurrence of DNOAP is quite rare. Second, PAD was confirmed as a complication, but risk factor analysis was not performed. The reason is that the control group was a DM foot patient with neuropathy, many of whom had already had PAD, and the neuropathy was already considered to be accompanied by microvascular complications. Finally, the severity of foot and ankle fracture was not considered. As mentioned earlier, since DNOAP occurs rarely it is hard to divide into sub-group. The association between foot and ankle fracture severity and BMD in DNOAP patients will also be analyzed when the case is more recruited.

Nevertheless, in this study, DNOAP patients with foot and ankle fractures confirmed a low femoral T score. Treatment goal of acute-phase DNOAP patients with foot and ankle fracture is immobilization and stability of protection destructive component. However, diabetic foot patients with neuropathy need constant BMD monitoring before these dystrophic lesions occur.

\section{CONCLUSIONS}

Low BMD shows greater incidence in foot and ankle fracture patients associated with neuropathic arthropathy. And femur T score can be a risk predictor of diabetic neuropathic arthropathy for diabetic foot patients.

\section{DECLARATIONS}

\section{Acknowledgments}

This work was supported by the National Research Foundation of Korea (NRF) grant funded by the Korea government (MSIT) (No. 2018R1C15042849).

\section{Ethics approval and consent to participate}

This study was approved by the Inje University Seoul Paik Hospital Research Ethics Committee (PAIK2018-08-007).

\section{Conflict of interest}

No potential conflict of interest relevant to this article was reported.

\section{ORCID}

Jae-Ho Cho

https://orcid.org/0000-0001-8680-4680

Tae-Hong Min

https://orcid.org/0000-0003-2832-1263

Dong-II Chun

https://orcid.org/0000-0001-7255-8984

Sung-Hun Won

https://orcid.org/0000-0002-5471-7432

Young Yi

\section{REFERENCES}

1. Charcot JM. Sur quelques arthropathies qui paraissent dépendre d'une lésion du cerveau ou de la moëlle épinière. Arch Physiol Norm Path 1868;1:161-78.

2. Frykberg RG, Mendeszoon E. Management of the diabetic Charcot foot. Diabetes Metab Res Rev 2000;16 Suppl 1:S5965.

3. Jordan WR. Neuritic manifestations in diabetes mellitus. Arch Intern Med 1936;57:307-66.

4. Young MJ, Marshall A, Adams JE, et al. Osteopenia, neurological dysfunction, and the development of Charcot neuroarthropathy. Diabetes Care 1995;18:34-8.

5. Hastings MK, Sinacore DR, Fielder FA, et al. Bone mineral density during total contact cast immobilization for a patient with neuropathic (Charcot) arthropathy. Phys Ther 2005;85:249-56.

6. Jackuliak P, Payer J. Osteoporosis, fractures, and diabetes. Int J Endocrinol 2014;2014:820615.

7. Clasen S. Is diabetic Charcot foot related to lower limb osteopaenia? Foot Ankle Surg 2000;6.

8. Kaynak G, Birsel O, Güven MF, et al. An overview of the Charcot foot pathophysiology. Diabet Foot Ankle 2013;4.

9. Gouveri E, Papanas N. Charcot osteoarthropathy in diabetes: A brief review with an emphasis on clinical practice. World J Diabetes 2011;2:59-65.

10. Jirkovská A, Kasalický P, Boucek P, et al. Calcaneal ultrasonometry in patients with Charcot osteoarthropathy and its relationship with densitometry in the lumbar spine and femoral neck and with markers of bone turnover. Diabet Med 2001;18:495-500.

11. Beeve AT, Brazill JM, Scheller EL. Peripheral Neuropathy as a Component of Skeletal Disease in Diabetes. Curr Osteoporos Rep 2019;17:256-69.

12. Rathinavelu S, Guidry-Elizondo C, Banu J. Molecular Modulation of Osteoblasts and Osteoclasts in Type 2 Diabetes. J Diabetes Res 2018;2018:6354787.

13. Jeffcoate WJ, Game F, Cavanagh PR. The role of proinflam- 
matory cytokines in the cause of neuropathic osteoarthropathy (acute Charcot foot) in diabetes. Lancet 2005;366: 2058-61.

14. Herbst SA, Jones KB, Saltzman CL. Pattern of diabetic neuropathic arthropathy associated with the peripheral bone mineral density. J Bone Joint Surg Br 2004;86:378-83.

15. SooHoo NF, Vyas R, Samimi D. Responsiveness of the foot function index, AOFAS clinical rating systems, and SF-36 after foot and ankle surgery. Foot Ankle Int 2006;27:930-4.

16. Kanis JA. Assessment of fracture risk and its application to screening for postmenopausal osteoporosis: synopsis of a WHO report. WHO Study Group. Osteoporos Int 1994;4: 368-81.

17. Hofbauer LC, Brueck CC, Singh SK, et al. Osteoporosis in patients with diabetes mellitus. J Bone Miner Res 2007;22: 1317-28.

18. Christensen TM, Bülow J, Simonsen $L$, et al. Bone mineral density in diabetes mellitus patients with and without a Charcot foot. Clin Physiol Funct Imaging 2010;30:130-4.

19. Jansen RB, Christensen TM, Bülow J, et al. Bone mineral density and markers of bone turnover and inflammation in diabetes patients with or without a Charcot foot: an 8.5-year prospective case-control study. J Diabetes Complications 2018;32:164-70.

20. Petrova NL, Foster AV, Edmonds ME. Calcaneal bone mineral density in patients with Charcot neuropathic osteoarthropathy: differences between Type 1 and Type 2 diabetes. Diabet Med 2005;22:756-61.

21. Mascarenhas JV, Jude EB. Pathogenesis and medical management of diabetic Charcot neuroarthropathy. Med Clin North Am 2013;97:857-72.

22. Trieb K. The Charcot foot: pathophysiology, diagnosis and classification. Bone Joint J 2016;98-b:1155-9.
23. Fauzi AA, Chung TY, Latif LA. Risk factors of diabetic foot Charcot arthropathy: a case-control study at a Malaysian tertiary care centre. Singapore Med J 2016;57:198-203.

24. Nóbrega MB, Aras R, Netto EM, et al. Risk factors for Charcot foot. Arch Endocrinol Metab 2015;59:226-30.

25. Hartemann-Heurtier A, Van GH, Grimaldi A. The Charcot foot. Lancet 2002;360:1776-9.

26. Nehring P, Mrozikiewicz-Rakowska B, Maroszek P, et al. Risk factors of charcot neuroarthropathy development in patients with type 2 diabetes. Exp Clin Endocrinol Diabetes 2014;122:31-4.

27. Pakarinen TK, Laine HJ, Honkonen SE, et al. Charcot arthropathy of the diabetic foot. Current concepts and review of 36 cases. Scand J Surg 2002;91:195-201.

28. Stuck RM, Sohn MW, Budiman-Mak E, et al. Charcot arthropathy risk elevation in the obese diabetic population. Am J Med 2008;121:1008-14.

29. Foltz KD, Fallat LM, Schwartz S. Usefulness of a brief assessment battery for early detection of Charcot foot deformity in patients with diabetes. J Foot Ankle Surg 2004; 43:87-92.

30. Jones J, Wolf S. Neuropathic shoulder arthropathy (Charcot joint) associated with syringomyelia. Neurology 1998;50: 825-7.

31. Sinacore DR, Hastings MK, Bohnert KL, et al. Inflammatory osteolysis in diabetic neuropathic (charcot) arthropathies of the foot. Phys Ther 2008;88:1399-407.

32. El Oraby HA, Abdelsalam MM, Eid YM, et al. Bone mineral density in type 2 diabetes patients with Charcot arthropathy. Curr Diabetes Rev 2019;15:395-401.

33. Park SY, Gong HS, Kim KM, et al. Korean guideline for the prevention and treatment of glucocorticoid-induced osteoporosis. J Bone Metab 2018;25:195-211. 
